\title{
A importância da avaliação otorrinolaringológica de pacientes com mucopolissacaridose
}

\section{The importance of the otorhinolaryngologic evaluation in mucopolysaccharidosis patients}

\author{
Cibele Gomes Bicalho', Morena Morais Rezende², Ana Maria Carrilho Moinhos Nogueira², Roberta Melo Calvoso Paulon ${ }^{3}$, \\ Angelina Xavier Acosta4.
}

1) Especialista em Otorrinolaringologia. Fellow em Otologia.

2) Especialista em Otorrinolaringologia. Professora substituta da disciplina de Otorrinolaringologia da Faculdade de Medicina da Bahia.

3) Graduada em Fonoaudiologia. Mestranda em Biotecnologia em Saúde e Medicina Investigativa pela Fundação Oswaldo Cruz - Fiocruz

4) Doutorado em Ciências Médicas. Professora da Disciplina de Pediatria da Faculdade de Medicina da Bahia Chefe do Serviço de Genética Médica do Complexo Hospitalar Universitário Professor Edgard Santos.

Instituição: Complexo Hospitalar Universitário Professor Edgard Santos. Salvador / BA - Brasil

Endereço para correspondência: Cibele Gomes Bicalho - Rua Augusto Viana S/N - Canela - Salvador/BA - Brasil - CEP: 40110-060 - E-mail: cibelebicalho@gmail.com Artigo recebido em 3 de Janeiro de 2011. Artigo aprovado em 7 de Abril de 2011

\section{RESUMO}

Introdução: Mucopolissacaridose (MPS) é um conjunto de doenças raras causadas pela deficiência de enzimas lisossômicas levando ao acúmulo de glicosaminoglanos (GAG) em órgãos e tecidos, responsáveis pelo quadro clínico multissistêmico, crônico e progressivo.

Objetivo: Descrever o perfil do exame clínico otorrinolaringológico de pacientes acompanhados no submetidos à Terapia de Reposição Enzimática (TRE) e propor um algoritmo de acompanhamento otorrinolaringológico para estes pacientes.

Método: Realizado estudo de série de casos incluindo 21 pacientes com MPS I, II e VI.

Resultados: As queixas otorrinolaringológicas mais frequentes foram obstrução nasal, roncos, respiração bucal. A presença de apneia foi relatada em 31\% dos casos. No exame físico a macroglossia foi a principal alteração (41\%) da orofaringoscopia. Na otoscopia, a retração da membrana timpânica esteve presente em $33 \%$ e a queixa de hipoacusia esteve presente em $45 \%$.

Conclusão: É importante avaliar as queixas, exame físico e o impacto das alterações associadas à respiração e audição produzidas pela MPS visando um melhor acompanhamento destes pacientes e a prevenção das alterações crônicas resultantes como a perda auditiva, distúrbios do sono e respiração bucal, promovendo assim uma melhor qualidade de vida destes pacientes.

Palavras-chave: glicosaminoglicanas. mucopolissacaridoses, otolaringologia.

\section{SUMMARY}

Introduction: Mucopolysaccharidosis (MPS) is a group of rare diseases caused by the deficit of lysosomal enzymes, causing an accumulation of glycosaminoglycans (GAG) in organs and tissues responsible for the multi-systemic clinical, chronic and progressive status.

Objective: Describe the profile of the otorhinolaryngologic clinical evaluation in patients submitted to Enzyme Replacement Therapy (ERT) and suggest an algorithm to otorhinolaryngolgically follow up with these patients.

Method: A study with a number of cases was performed, including 21 patients having MPS I, II and VI.

Results: The most common otorhinolaryngological complaints were nasal obstruction, snore, and mouth-breathing. Apnea was reported in $31 \%$ of the cases. In the physical examination, macroglossia was the main alteration (41\%) of oral pharyngoscopy. At otoscopy, the retraction of the tympanic membrane was found in $33 \%$ and hearing loss was found in $45 \%$.

Conclusion: It is important to evaluate the complaints, physical examination and the impact of alterations associated with MPSproduced breathing and hearing, for purposes of better following up with these patients and preventing consequential chronic alterations such as hearing loss, sleep disorder, and mouth breathing, thus, enhancing these patients' quality of life.

Keywords: glycosaminoglycans, mucopolysaccharidosis, otolaryngology. 


\section{INTRODUÇÃO}

Mucopolissacaridose (MPS) é um conjunto de doenças raras causadas pela deficiência de enzimas lisossômicas levando ao acúmulo de glicosaminoglanos (GAG) em órgãos e tecidos, responsáveis pelo quadro clínico multissistêmico, crônico e progressivo. Já foram identificados 11 defeitos enzimáticos que causam 7 tipos diferentes de MPS. O tratamento das mucopolissacaridoses era inicialmente sintomático e paliativo, baseado em uma equipe multidisciplinar, com a participação de diversas especialidades médicas como cardiologia, pneumologia, anestesia, ortopedia, otorrinolaringologia, oftalmologia, neurocirurgia, entre outros, bem como, profissionais da fisioterapia, terapia ocupacional, psicologia, e fonoaudiologia (1). Atualmente, além da equipe multidisciplinar, já há uma terapia específica para os tipos I, II e VI, a terapia de reposição enzimática (TRE) que consiste na administração periódica, por via venosa, da enzima específica deficiente no paciente. Este tratamento vem proporcionando bons resultados na melhora clínica e da qualidade de vida (2).

A MPS I, como a maioria das doenças lisossômicas, é herdada de modo autossômico recessivo e tem uma incidência de aproximadamente 1 em 100 mil nascidos vivos (3). Os fenótipos relativos a MPS I podem se apresentar como a Síndrome de Hurler, Hurler-Scheie e Scheie. Sendo que a síndrome de Hurler é a forma mais grave da doença e a síndrome de Scheie, a forma mais atenuada.

A MPS II (Síndrome de Hunter) apresenta uma incidência de aproximadamente 0,31 a 0,71 por 100 mil nascidos vivos $(4,5,6)$ sendo encontrada quase exclusivamente em meninos, por se tratar de doença genética ligada ao cromossomo X.

A mucopolissacaridose VI (MPS VI ou Síndrome de Maroteaux-Lamy) é uma doença genética rara, autossômica recessiva, causada pela deficiência da enzima $\mathrm{N}$-acetilgalactosamina-4-sulfatase, ou arilsulfatase B. A incidência estimada para a MPS VI é de 0,23 por 100 mil nascidos vivos.

O diagnostico de MPS pode ser realizado de acordo com suspeita clinica, mensuração de GAG urinário confirmação através de avaliação enzimática. Na maioria dos casos a doença se apresenta na infância e sintomas clínicos estão ausentes ao nascimento. Os pacientes com MPS apresentam uma variabilidade ampla de sintomas multissistêmicos, de curso crônico e progressivo, sendo acometidos principalmente o sistema esquelético e cardiopulmonar, a córnea, a pele, o fígado, o baço, o cérebro e as meninges.
As manifestações clínicas otorrinolaringológicas mais comuns são as anomalias respiratórias e infecções repetidas de via aérea superior (VAS) devido a macroglossia, hipertrofia de adenoides e amígdalas, às alterações esqueléticas da mandíbula e coluna cervical, apneia do sono, otites de repetição e hipoacusia. A avaliação otorrinolaringológica pode preceder o diagnostico da síndrome em cerca de 30\% dos casos (7).

O conhecimento do conjunto de manifestações otorrinolaringológicas deve ser bem estudado para auxiliar no diagnóstico precoce da síndrome bem como para o tratamento específico direcionado que leve a redução das comorbidades e melhora na qualidade de vida. No entanto, são escassos na literatura estudos envolvendo este grupo de pacientes.

Este é um estudo de corte transversal com o objetivo de descrever o perfil do exame clínico otorrinolaringológico de pacientes submetidos a TRE.

\section{MÉTODO}

Foi realizada avaliação otorrinolaringológica de 21 pacientes com MPS e em uso de TRE.

Entre os pacientes avaliados, 12 possuem MPS tipo VI, 6 do tipo II e 3 do tipo I. Em relação aos pacientes com MPS tipo I, 1 tem forma Hurler, 1 Hurler Scheie e 1 Scheie. Entre os portadores de MPS I e VI, 12 eram do sexo masculino. Todos os pacientes com MPS II são do sexo masculino. A idade variou de 1 a 20 anos, sendo que a media de idade foi 9,14 anos.

Foram incluídos todos os pacientes acompanhados no hospital de estudo, em uso de TRE. A duração da terapia variou de 7 a 60 meses com média de 25,71 meses.

Foram realizadas entrevistas com os responsáveis e avaliação física dos pacientes por médicos otorrinolaringologistas.

Este estudo tem aprovação pelo Comitê de Ética sob o parecer numero 010/2009.

\section{RESULTADOS}

Cerca de $66 \%$ dos pacientes apresentavam queixa de obstrução nasal, que se apresentou de maneira continua em 38\% dos pacientes e graduada como acentuada em $30 \%$ dos casos. A presença de roncos foi relatada em $71 \%$ dos pacientes, destes $50 \%$ apresentavam o sintoma diariamente e a pausa respiratória foi relatada em 31\%\% 
Tabela I. Frequência das queixas otorrinolaringológicas de acordo com o tipo de MPS e total de pacientes avaliados.

\begin{tabular}{lllll}
\hline & MPSI & MPS II & MPSVI & Total \\
\hline Obstrução nasal & $66 \%$ & $66 \%$ & $66 \%$ & $66 \%$ \\
Roncos & $66 \%$ & $100 \%$ & $58 \%$ & $71 \%$ \\
Respiração bucal & $100 \%$ & $66 \%$ & $75 \%$ & $76 \%$ \\
apneia & $\ldots$ & $\ldots$ & $71 \%$ & $31 \%$ \\
Rinorreia & $100 \%$ & $33 \%$ & $50 \%$ & $52 \%$ \\
OMAde repetição & $\ldots$ & $50 \%$ & $41 \%$ & $38 \%$ \\
RSAde repetição & $\ldots$ & $\ldots$ & $1 \%$ & $<1 \%$ \\
Hipoacusia & $33 \%$ & $80 \%$ & $50 \%$ & $45 \%$ \\
\hline
\end{tabular}

Legenda: OMA - Otite media aguda RSA - rinossinusite de repetição.

dos casos. Os roncos tiveram maior frequência nos pacientes com MPS I e II, sendo que os pacientes com MPS tipo VI com queixa de roncos, os apresentavam com maior intensidade e mais frequente associação com apneia do sono. A respiração se apresentou predominantemente bucal em $76 \%$ dos pacientes. Metade dos pacientes apresentaram queixa de rinorreia, na maioria dos casos de maneira intermitente $(77,8 \%)$ e amarelada $(55 \%)$.

O principal tipo de infecção de VAS foi à otite media aguda. A hipoacusia foi um sintoma relatado em apenas 9 casos (45\%). Em relação aos tipos de MPS, pode-se comparar a frequência dos sintomas na Tabela 1.

Quanto ao exame físico, a retração de MT foi a mais frequente alteração na otoscopia (33\%), sendo que 30\% apresentavam otoscopia normal no momento da avaliação (Tabela 2). Na rinoscopia anterior a maioria dos pacientes não apresentavam alterações.

$\mathrm{Na}$ orofaringoscopia as amígdalas não foram visualizadas em $60 \%$ dos casos, e nos casos com boa visualização a maioria se apresentavam eutroficas. O grau de hipertrofia amigdaliana e a possibilidade de visualização da orofaringe variou entre os tipos de MPS (Gráfico 1).

Verificado ainda macroglossia em quase $100 \%$ dos casos, acentuada em 40\%. Os pacientes com MPS II e VI apresentaram macroglossia mais acentuada e todos os pacientes com o tipo I apresentaram apenas leve macroglossia (Gráfico 2).

\section{DISCUSSÃO}

O comprometimento de VAS é um dos mais desafiadores aspectos no tratamento destes pacientes. As alterações de VAS incluem macroglossia, hipertrofia adenoamigdaliana, deposito de GAG em faringe e laringe.
Tabela 2.

\begin{tabular}{llllll}
\hline & & MPSI & MPS II & MPSVI & Total \\
\hline normal & OD & $66 \%$ & $33 \%$ & $17 \%$ & $30 \%$ \\
& OE & $66 \%$ & $33 \%$ & $17 \%$ & $30 \%$ \\
Cerume & OD & $\ldots$ & $\ldots$ & $17 \%$ & $1 \%$ \\
& OE & $\ldots$ & $17 \%$ & $17 \%$ & $14 \%$ \\
Retração & OD & $33 \%$ & $17 \%$ & $42 \%$ & $33 \%$ \\
& OE & $33 \%$ & $17 \%$ & $42 \%$ & $33 \%$ \\
Opacificação & OD & $\ldots$ & $33 \%$ & $25 \%$ & $24 \%$ \\
& OE & $\ldots$ & $17 \%$ & $25 \%$ & $19 \%$ \\
Estenose de CAE & OD & $\ldots$ & $17 \%$ & $\ldots$ & $<1 \%$ \\
& OE & $\ldots$ & $17 \%$ & $\ldots$ & $<1 \%$ \\
\hline
\end{tabular}

Legenda: OD - orelha direita OE - orelha esquerda CAE conduto auditivo externo.

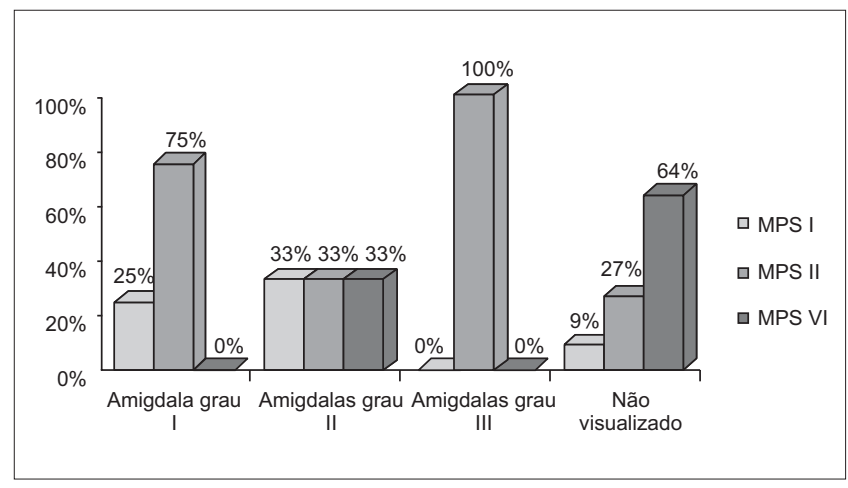

Gráfico 1. Comparação da avaliação da orofaringe em relação a MPS I, II, VI.

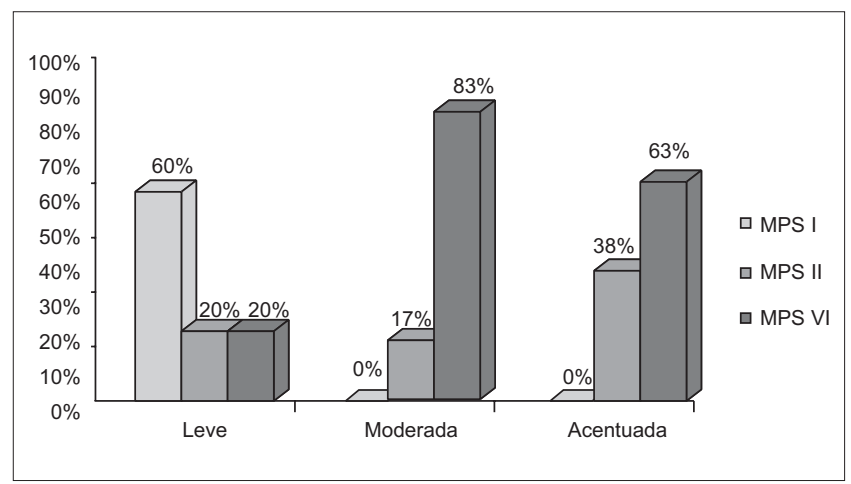

Gráfico 2. Comparação do grau de macroglossia na MPS I, II, VI.

Em avaliação de 27 pacientes com MPS cerca de 70\% apresentaram obstrução clinica de vias aéreas superiores (8), de acordo com a avaliação realizada nos nossos pacientes. As alterações craniofaciais aliadas à instabilidade de cabeça e pescoço os tornam de difícil intubação com alto risco anestésico. $\mathrm{Na}$ avaliação de 89 procedimentos anestesicos, dificuldade de entubação foi encontrada em $25 \%$ dos casos, sendo que os pacientes com síndrome de 
Hurler apresentaram o dobro de dificuldades (9). Nesta avaliação em quase $60 \%$ dos casos houve dificuldade na avaliação de lojas amigdalianas ao exame clinico, esta dificuldade provavelmente ocorreu devido a macroglossia, e avaliação devera ser complementada pela laringoscopia. A decisão quanto ao manejo da hipertrofia adenoamigdaliana deverá basear-se na clinica e polissonografia devido ao risco anestésico destes pacientes.

A prevalência de alterações audiológicas em pacientes com MPS ainda não é conhecida. Esses pacientes apresentam comprometimento neurológico com grande frequência, sendo difícil uma avaliação audiométrica satisfatória. Sabe-se que a hipoacusia é uma manifestação frequente nesses pacientes, neste estudo, foi relatada por familiares em $40 \%$ dos pacientes. Este número poderá se apresentar ainda maior após realização de audiometria.

WolD et al, ao avaliar pacientes com MPS I,II e VI, mostraram $78 \%$ de perda auditiva através de avaliação audiométrica. Destes, $71 \%$ apresentaram perda auditiva mista, $14 \%$ sensorioneural e $14 \%$ condutiva (7). Avaliação histológica do osso temporal sugere haver uma interferência no desenvolvimento em torno de 5 a 6 meses de idade gestacional; havendo diminuição da pneumatização da mastoide, persistência de cápsula ótica cartilaginosa adjacente ao canal semicircular posterior e aumento da artéria subarcuata (10). As perdas auditivas condutivas estão relacionadas à presença de efusão e comprometimento ossicular em orelha media (11). Nesta avaliação não foi verificada clinicamente presença de efusão em orelha media destes pacientes, o que pode ser explicado pela idade de avaliação, pelo uso de terapia de reposição enzimática, ou pela opacificação da membrana timpânica, havendo necessidade de acompanhamento a longo prazo e comparação com pacientes que não estejam em uso da terapia.

A etiologia das perdas neurosensoriais permanecem obscuras, sendo sugeridas possibilidade de compressão nervosa por hiperplasia de aracnoide e destruição axonal no gânglio espiral (10).

Estudos prévios (11) mostram a perda auditiva mais frequente nos pacientes portadores de MPS I e II, dados estes que diferem dos apresentados nesta avaliação, esta divergência pode ter acontecido pelo maior numero de pacientes com MPS VI incluídos neste estudo.

Em avaliação retrospectiva de 09 pacientes portadores de MPS I,II,III com media de idade de 3 anos, foi verificado que todos precisaram ser submetidos colocação de TV devido à otite media secretora/otite media aguda de repetição, metade destes pacientes foram submetido à conduta conservadora inicialmente, sem resolução; nos

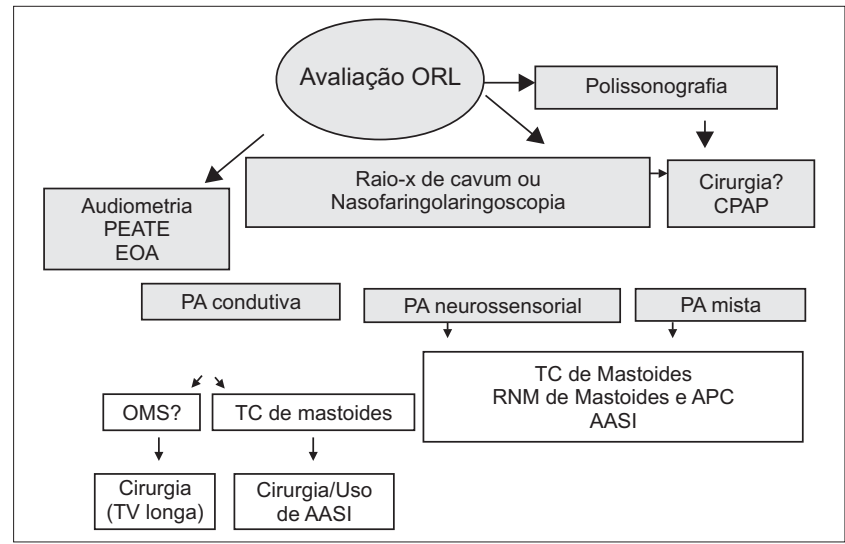

Figura 1. PEATE - Potencial evocado auditivo do tronco cerebral EOA - Emissões Otoacusticas TC - Tomografia Computadorizada RNM - Ressonância Magnética TV - tubo de ventilação CPAP -Pressão positiva continua nas vias aéreas.

pacientes que optaram pelo tubo de ventilação de curta permanência, necessitaram repetir o processo, o autor recomenda que seja escolhido o tubo de longa permanência (12).

No nosso serviço esta sendo realizado o seguinte fluxograma delineado na Figura 1 desde avaliação inicial até a proposta de diagnostico e intervenção posterior. Os dados presentes neste artigo representam a avaliação otorrinolaringológica inicial destes pacientes que estão sendo submetidos à avaliação auditiva e de via aérea superior mais detalhadas.

\section{CONCLUSÃO}

O acompanhamento destes pacientes exige colaboração de equipe multidisciplinar, e a documentação da evolução da patologia pode ser realizada com base em protocolos de acompanhamento e diagnostico. A avaliação e o acompanhamento Otorrinolaringológico devem ser precoces a fim de prevenir maior impacto sistêmicos das alterações de vias aéreas superiores e comprometimento auditivo irreversível.

\section{REFERÊNCIAS BIBLIOGRÁFICAS}

1. Pastores GM, Arn P, Beck M, Clarke JT, Guffon N, Kaplan $\mathrm{P}$, et al. The MPS I registry: design, methodology, and early findings of a global disease registry for monitoring patients with Mucopolysaccharidosis Type I. Mol Genet Metab. 2007, 91(1):37-47.

2. Giugliani R, Federhen A, Munoz Rojas MV, Vieira TA, Artigalas O, Pinto LL, et al. [Enzyme replacement therapy 
for mucopolysaccharidoses I, II and VI: recommendations from a group of Brazilian F experts.]. Rev Assoc Med Bras. 2010, 56(3):271-7.

3. Meikle PJ, HopwoodJJ, Clague AE, Carey WF. Prevalence of lysosomal storage disorders. JAMA. 1999 Jan 20, 281(3):249-54.

4. Baehner F, Schmiedeskamp C, Krummenauer F, Miebach E, Bajbouj M, Whybra C, et al. Cumulative incidence rates of the mucopolysaccharidoses in Germany. J Inherit Metab Dis. 2005, 28(6):1011-7.

5. Nelson J, Crowhurst J, Carey B, Greed L. Incidence of the mucopolysaccharidoses in Western Australia. Am J Med Genet A. 2003 Dec 15, 123A(3):310-3.

6. Nelson J. Incidence of the mucopolysaccharidoses in Northern Ireland. Hum Genet. 1997, 101(3):355-8.

7. Wold SM, Derkay CS, Darrow DH, Proud V. Role of the pediatric otolaryngologist in diagnosis and management of children with mucopolysaccharidoses. Int J Pediatr Otorhinolaryngol. 2010, 74(1):27-31.
8. Andrea H.Yeung M, Morton J.Cowan M, Biljana Horn M, Kristina W. Rosbe M. Airway Management in Children With Mucopolysaccharidoses. Arch Otolaryngol Head Neck Surg. 2009, 135(1):73-79.

9. Walker RW DMMPWJE. Anaesthesia and mucopolysaccharidoses. A review of airway problems in children. Anaesthesia. 2010, 49(12):1078-1084.

10. Hayes E, Babin R, Platz C. The otologic manifestations of mucopolysaccharidoses. Am J Otol. 1980, 2(2):65-9.

11. Papsin BC, Vellodi A, Bailey CM, Ratcliffe PC, Leighton SE. Otologic and laryngologic manifestations of mucopolysaccharidoses after bone marrow transplantation. Otolaryngol Head Neck Surg. 1998, 118(1):30-6.

12. Motamed M, Thorne S, Narula A. Treatment of otitis media with effusion in children with mucopolysaccharidoses. Int J Pediatr Otorhinolaryngol. 2000, 53(2):121-4. 